\title{
REDUCTION OF PULSE DISTORTION IN \\ TRAVELLING WAVE SEMICONDUCTOR OPTICAL AMPLIFIDRS
}

\author{
M.J.Soneira, J.F.Gonzalez, S.Ruiz-Moreno \& J.Guitart \\ Dpto. Teoría de la Señal y Comunicaciones. ETSIT-BARCELONA, UPC \\ c/Jorge Girona Salgado s/n. 08034 BARCELONA. SPAIN.
}

\begin{abstract}
The nonlinear phenomenon inherent in travelling wave semiconductor optical amplifiers can produce several harmful effects in transmission systems such as pulse distortion in multi-Gbit/s intensity-modulation systems. In this communication, is presented a theoretical investigation of the possibility of reducing the gain saturation during the pulse amplification process by means of the compensation of the carrier density variations. This should be very useful in many optical systems and, specially, in high speed communication systems.
\end{abstract}

\section{INTRODUCTION}

Travelling wave semiconductor optical amplifiers (TWOA) are emerging as practical components for use in optical communications systems, using both direct and coherent detection. Several works [1],[2] have shown that they posses many advantages, being its high gain over a very wide bandwidth one of the most outstanding characteristic. Because of its large bandwidth TWOA are specially suitable to amplify narrow optical pulse [3]. The device gain can be significantly reduced during the pulse propagation and, in consequence, the trailing pulse edge can receive less gain than the leading edge causing the pulse distortion. This is due to the gain saturation from decrease of carrier density.

On the other hand, in a high bit rate transmission system is thinkable that the time interval between two consecutive signals is comparable to or even smaller than the carrier lifetime of the device. As a consequence, the carrier density will not be enough time to recover. Clearly, the gain experienced by each individual pulse will be different from each other and dependent on the foregoing signals. In this communication we study the possibility of reducing the variations of the carrier density and so the saturation gain under dynamic conditions. The method proposed to reduce the saturation gain takes into account the propagation coordinate because the carrier density diminution is larger at the end of the amplifier. In Section 2 we stablish both the basic equations which govern the dynamics of the amplification process and the theoretical base of the compensation scheme of the carrier density. Some results are presented in Section 3, where we discuss the effects of compensation on the amplified pulse and on the carrier density. The main conclusions are drawn in Section 4.

\section{NONLINEAR MODEL OF A TWOA}

\subsection{Basic Equations}

In the study of pulse propagation in TWOA, the amplifier is modeled as a set of two level systems with transition energies extending over the whole range of the conduction and valence bands. The basic equations which govern pulse propagation in semiconductor optical amplifier are

$$
\begin{gathered}
\frac{d P(z, t)}{d z}=\left[G_{p}(N, P)-L_{p}\right] \cdot P(z, t) \\
\frac{d \phi(z, t)}{d z}=F(N, P) \\
\frac{d N(z, t)}{d t}=\frac{N B(z, t)-N(z, t)}{\tau s}-\frac{G_{P}(N, P) \cdot P(z, t)}{h v w \cdot d}
\end{gathered}
$$

where (1) and (2) come from the wave equation and they give the longitudinal variation of power, $P$, and phase, $\phi$, of the pulse. $G_{p}$ and $L_{p}$ are the power gain and the losses in the medium respectively, $F$ is the phase gain and $\mathrm{N}$ is the carrier density. The equation (3) is the carrier density rate equation which govern the carrier density within the active region. NB 
is the carrier density due to bias current, $\tau$ s is the carrier lifetime, $h$ is the Plank constant, $v$ is the optical frequency and $w \cdot d$ is the active region cross area. The gain functions can be expressed as

$$
\begin{gathered}
G_{p}(N, P)=\Gamma G(N)\left[1-K_{p}(P)\right] \\
F(N, P)=-\frac{\alpha}{2} \cdot G(N)[1-K F(P)]
\end{gathered}
$$

where $\Gamma$ is the confinement factor. $G(N)$ contains the dependence with the carrier density, which is represented by a polynomical approximation given by $G(N)=\Sigma A_{1} N^{1}$ with $\mathrm{i}=1 . .4$. $\mathrm{K}_{\mathrm{p}}(\mathrm{P})$ and $\mathrm{K}_{\mathrm{F}}(\mathrm{P})$ are the gain suppresion functions which are defined [4]

$$
K_{p}(P)=\frac{P / P_{s}}{\sqrt{1+P / P_{s}}\left(\sqrt{1+P / P_{s}}+1\right)}
$$

$$
\left.K F(P)=\frac{\beta}{\alpha} \cdot \frac{P / P_{s}}{\left(\sqrt{1+P / P_{s}}+1\right.}\right)
$$

being $\beta$ and $\alpha$ phenomenological constants and Ps a normalized power which depends on the semiconductor medium. The parameters values used in the simulations are summarized in Table 1 .

\begin{tabular}{|c|c|}
\hline wd & $0.3 \mu \mathrm{m}^{2}$ \\
$\mathrm{~L}$ & $300 \mu \mathrm{m}$ \\
$\lambda$ & $1.55 \mu \mathrm{m}$ \\
$\Gamma$ & 0.3 \\
$\mathrm{A0}$ & $-1.92 \cdot 10^{5}$ \\
$\mathrm{A1}$ & $2.42 \cdot 10^{5}$ \\
$\mathrm{~A} 2$ & $-8.47 \cdot 10^{5}$ \\
$\mathrm{A3}$ & $1.55 \cdot 10^{4}$ \\
$\mathrm{~A} 4$ & $-1.06 \cdot 10^{3}$ \\
$\mathrm{Lp}$ & $4000 \mathrm{~m}^{-1}$ \\
$\tau \mathrm{s}$ & $0.3 \mathrm{n} \mathrm{s}$ \\
$\alpha$ & 5.0 \\
$\beta$ & 4.5 \\
Ps & $420 \mathrm{~mW}^{24}$ \\
NBo & $2.5 \cdot 10^{-3} \mathrm{~m}^{3}$ \\
\hline
\end{tabular}

Table I

\subsection{Theoretical compensation of} nonlinearity in TWOA

In the same way than [5], in order to compensate the diminution of the carrier density we have separated NB into two terms

$$
\mathrm{NB}(z, t)=\mathrm{NBO}+\mathrm{NBC}(z, t)
$$

the first term is constant and represents the pumping carrier density necessary to obtain the small signal gain, that is,

$$
\mathrm{Go}_{0}=\left.\mathrm{G}_{\mathrm{p}}\right|_{\mathrm{N}=\mathrm{NBO}, \mathrm{P}=0}
$$

and the second term is the compensation term defined by

$$
\operatorname{NBC}(z, t)=\frac{\tau_{z} \cdot G_{p}(N)}{h v w \cdot d} \cdot P(z, t)
$$

with this, the solution of equation (3) can be approximate as $\mathrm{N} \cong N B o$ when pulse width is much shorter than the carrier lifetime. But equation (10) presuppose to know the gain and the optical power at each point $z$ of the amplifier which is unthinkable. However, if we assume that exits an ideal compensation the gain can approximate as

and so,

$$
\mathrm{Gp}(N) \cong \mathrm{Go}_{0}
$$

$$
\mathrm{P}(z, t) \cong \operatorname{PIN}(t) \cdot e^{\left(\mathrm{G}_{0}-\mathrm{Lp}\right) z}
$$

that is, (11) and (12) are reasonable approximations as of ten as $\mathrm{N}(z, t) \simeq \mathrm{NBo}$. Then, substituting in equation (10) we obtain

$$
\operatorname{NBC}(z, t)=\frac{\tau_{\mathrm{s}} \cdot \mathrm{Go}_{0}}{h v \mathrm{w}_{\mathrm{d}}} \cdot e^{\left(\mathrm{Go}-\mathrm{Lp}_{\mathrm{p}}\right) z} \cdot \operatorname{PIN}(t)
$$

therefore, the compensation function depends on the input optical signal and the $z$-coordinate by a exponential function. It is possible to approximate this function by sections dividing the amplifier into a number $m$ of equal sections. In each of these the pumping carrier density takes the mean value of $\operatorname{NBc}(z, t)$, then we can obtain

$$
\begin{aligned}
\operatorname{NBC}(i, t)= & \frac{m \cdot \tau_{s}}{h v \cdot d \cdot d} \cdot \frac{G_{0}}{G_{0}-L_{p}} \cdot e^{i\left(G_{0}-L_{p}\right) \frac{L}{m}} \\
& \cdot\left(1-e^{\left(G_{0}-L_{p}\right)} \frac{L_{m}}{m}\right) \cdot \operatorname{PIN}(t)
\end{aligned}
$$

where $i=1, \ldots, m$ is the section number and $L$ is the amplifier length. If $m=1$ (only one section) the result is the same that [5] doing $L_{p}$ « Go. So then the scheme proposed to compensate the carrier density is drawn in figure 1.

\section{RESULTS AND DISCUSSION}

The set of equations (1), (2) and (3) can 


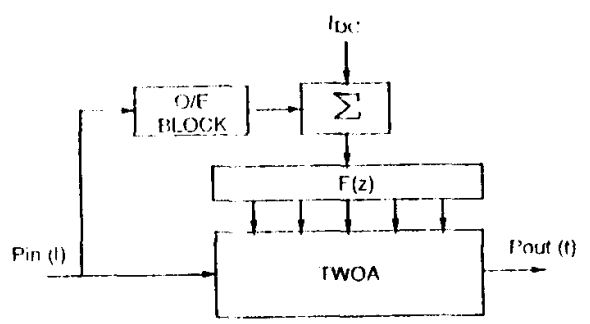

Figure 1: Compensation scheme.

not be solved analytically and, in consequence, is necessary its numerical solution. In the simulations we have considered a Gaussian pulse for which

$$
\operatorname{PIN}(t)=\frac{E_{1 \mathrm{n}}}{t 0 \sqrt{\pi}} \cdot e^{-\left(t / t_{0}\right)^{2}}
$$

where Eln is the input pulse energy and to is related to the full width at half maximum (FWHM) by $t_{p}=1.665$ to. In figure 2 is represented the carrier density as a function of the propagation coordinate $z$ for the pulse peak when there is no compensation, curve (c), and when there is theoretical compensation (13), curves (b) and (a). The difference between these last curves is that (b) does not take into account the supression gain terms in contrast to (a) where they are taken into account. Of course, when there is no compensation the carrier density decrease along amplifier length when pulse is passing Nevertheless, when compensation is operating the carrier density is aproximately constant if the gain supression terms are not taking into acount $\left(\mathrm{K}_{\mathrm{p}}(\mathrm{P})=\mathrm{K}_{F}(\mathrm{P})=0\right)$. On the contrary, when they are taking into account exits a light increment at the end of the amplifier. The value of carrier density given by (13) is larger than the value given by (10) because the first does not look at the gain disminution due to optical power in the medium (supression phenomenon).

In figure 3 is shown the evolution of carrier density along amplifier length when there is compensation considering the theoretical case (curve (a)), only one section (curve (b)) and four sections (curve (c)). We can observe that the average of the carrier density in each section is approximately constant and equal to the carrier density obtained in the theoretical compensation case. This is the main reason from what the shape and phase of the amplified pulse are independent of the

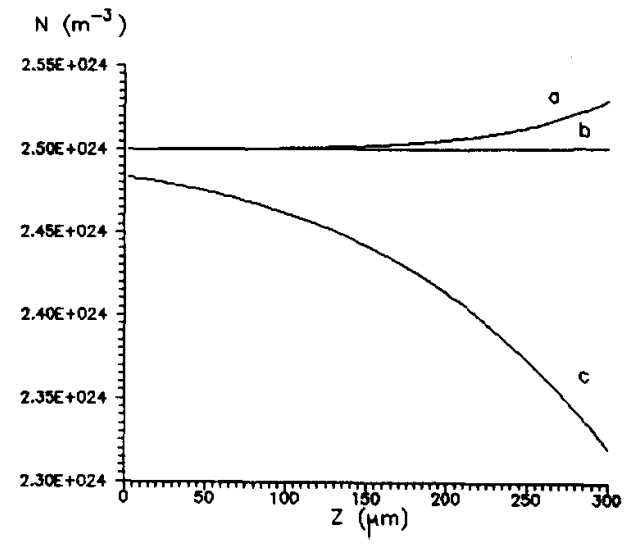

Figure 2: Theoretical compensation.

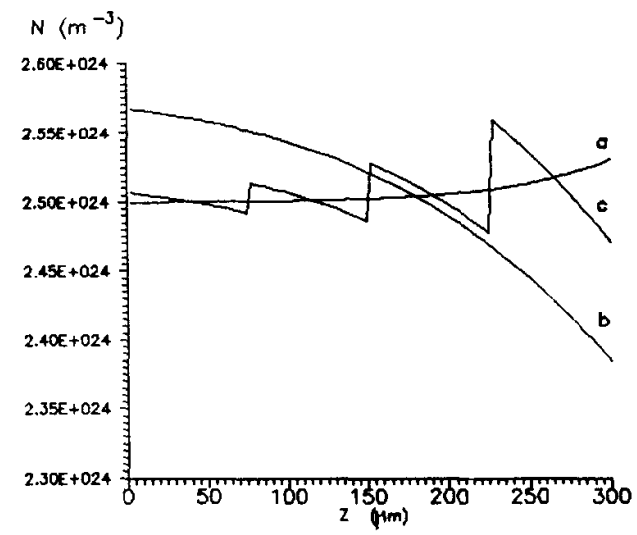

Figure 3: Evolution of carrier density for different sections.

sections number used as we will see in figures 4 and 5 . However, the section number has influence on the final level achieved to the carrier density and, therefore, on the recuperation time to initial state (before the pulse amplification) of the amplifier. If this time decreases, the separation between two consecutive pulses can decrease, being the gain of each individual pulse the same.

The input pulse phase represented by equation (15) is equal to zero, nevertheless will be modified during the amplification process. The diference between the instantaneous frequency and the optical frequency can be obtained as

$$
\Delta \mathrm{frec}=-\frac{1}{2 \pi} \frac{\mathrm{d} \phi}{\mathrm{dt}}
$$




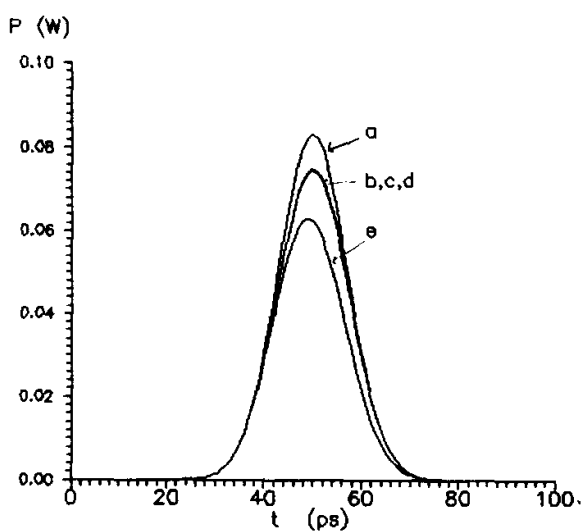

Figure 4: Amplified pulse power.

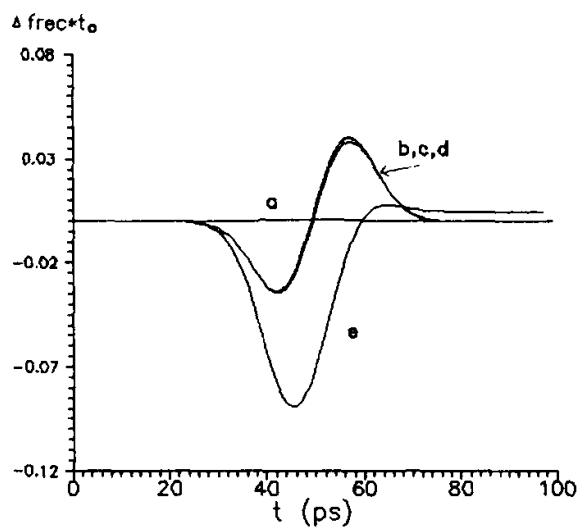

Figure 5: Output frequency displacement.

In figures 4 and 5 are shown the shape and frequency displacement, $\Delta \mathrm{frec}$, of de amplified pulse, respectively. The curves (e) represent the situation where there is no compensation and the others are for the compensation situation. The curves (a) and (b) represent the theoretical compensation without and with supression gain, and (c) and (d) represent the compensation case with supression gain for one and four sections respectively. The figure 4 shows as the amplified pulse without compensation is lightly assymetric (the pulse maximum shifts to the leading pulse edge) as a consequence that the trailing pulse edge receive lower gain than the trailing edge. In a compensation situation (curves (b),(c) and (d)) the shape of amplified pulse is independent of the number of amplifier sections. This is due to each section has the same average gain along the amplifier length. In figure 5 can be observed that whithout compensation the frequency displacement increases almost linearly over the central part of the pulse. Such a linear characteristic implies that the pulse can be compressed in a dispersive medium. Like the shape, the frequency displacement is independent of the number of sections. On the other hand, the displacement maximum is lower in a compensation situation. Noted that in a compensation situation without supression (curve (a)) $\Delta$ frec is almost equal to the input one. This result confirms that the compensation of the carrier density diminution is more effective at least important is the supression phenomenon.

\section{CONCLUSIONS}

In this communication we have presented theoretical results relate to dynamic compensation of the saturation gain in semiconductor optical amplifiers. The method to reduce the saturation gain consists of a inhomogeneous pump of the amplifier. This inhomogeneity can be achieved using a multisegment amplifier. The obtained results show that when the number of sections increase, the time interval between two consecutives pulses can decrease being dynamic gain equal to each individual pulse.

On the other hand, this compensation method is valid for low variations of the carrier density in relation to the injected carrier density (NBO). Under this conditions, the dynamic gain is close to small signal gain of the amplifier.

\section{REFERENCES}

[1] J.C.Simon, "Semiconductor Laser Amplifier for Single Mode Optical", J. Opt.Comm., v.4, no.2, 1983.

[2] T. Saitoh \& T. Mukai, "1,5 $\mu \mathrm{m}$ GaInAsP Travelling-Wave Semiconductor Lasers Amplifiers", IEEE J.Q.Electron., v.23, no. 6, 1987.

13] I.E.Marshall, et al."Picosecond Pulse Response of a Travelling-Wave Optical Amplifier", Elec.Lett.,v.24,no.16,1987

[4] G.P.Agrawal,"Spectral Hole-Burning and Gain Saturation in Semiconductor Lasers: Strong-Signal Theory", J.Applied Physics, v. 63, no.4, 1988.

[5] A.A.M.Saleh, et al., "Compensation of nonlinearity in Sc.optical amplifiers" El.Lett., vol.24, no.15, 1988. 